\title{
Shape transition in the neutron-rich $Y$ nuclei and its evolution across the isotopic chain
}

\author{
Eukasz W. Iskra ${ }^{1,2, *}$, Bogdan Fornal ${ }^{2}$, Silvia Leoni ${ }^{1,3}$, Caterina M ichelagnoli ${ }^{4}$, Simone B ottoni ${ }^{1,3}$, Natalia Cieplicka- \\ Oryńczak ${ }^{2}$, Michael J entschel ${ }^{4}$, Felix K andzia4, Yung Hee Kim², Ulli Köster $^{4}$, and Carlotta Porzio ${ }^{3}$
}

IINFN sezione di M ilano via Celoria 16, 20133, M ilano, Italy

Institute of Nuclear Physics, PA N, 31-342 Krakow, Poland

${ }^{3}$ Dipartimento di Fisica, Università degli Studi di Milano, I-20133 M ilano,

${ }^{4} \mathrm{LL}$, 71 A venue des M artyrs, 38042 Grenoble CEDEX 9, France

\begin{abstract}
The evolution of the deformation across the $Y$ isotopic chain, in the vicinity of $N=60$ boundary, has been studied using gamma spectroscopy methods. The nuclei of interest have been produced by neutron induced fission of ${ }^{235} \mathrm{U}$ and ${ }^{241}$ Pu targets, during two experimental campaigns named EXILL and FIPPS at the Institute $L$ aue- $L$ angevin in Grenoble. The emitted gamma rays have been collected by $H P G e$ and $L a B r_{3}$ detectors providing the identification of the high-spin levels in the ${ }^{94} \mathrm{Y}$ and ${ }^{96} \mathrm{Y}$ isotopes up to $6 \mathrm{MeV}$ excitation energy, as well as information about the half-lives of the in-band states in the ${ }^{98} Y$ nucleus. The obtained results shed new light on the onset of deformation in the neutron-rich $Y$ isotopes and, in general, in the $\mathrm{A} \approx 100$ region.
\end{abstract}

\section{Introduction}

The region around $Z=40$ and $N=60$ is especially important to test the mechanism of creation of deformed structures, as well as to study its evolution across specific isotopic chains. This is due to the sudden onset of deformation at the ground states in the nuclei with $\mathrm{N}$ $\geq 60$, which is reported as the most dramatic shape change in the whole nuclear chart [1]. For lighter systems, the ground levels have shown spherical character, while rotational bands are observed at higher excitation energy. In the case of $\mathrm{Zr}(Z=40)$ and $\mathrm{Sr}(Z=$ 38) isotopes, the coexistence of such structures has already been observed at $\mathrm{N}=58,59$ [2]. However, the parameters of deformation $\beta$, extracted from the lifetime of in-band states, in the ${ }^{96,97} \mathrm{Sr}$ and ${ }^{98,99} \mathrm{Zr}$ nuclei [2] are significantly smaller than for ground state rotational bands at $\mathrm{N} \geq 60$. In the case of the $\mathrm{Y}$ isotopic chain, the experimental information about the deformation size of the rotational band was far less complete. In a very recent study of T. W. Hagen et al., [3], the half-lives of the several deformed states in ${ }^{99} \mathrm{Y}$ and ${ }^{101} \mathrm{Y}$ have been measured and a deformation parameter $\beta \sim 0.4$ has been obtained. A similar value was measured by the authors of this collaboration for the rotational band in ${ }^{98} \mathrm{Y}(\mathrm{N}=$ 59) isotope, which was briefly reported in Ref. [4]. The observation of strongly deformed rotational band in the ${ }^{98} \mathrm{Y}$ nucleus raises the question if similar bands are present also in lighter isotopes, further away from the $\mathrm{N}$ $=60$ boundary. The searching of such structures in the
${ }^{94} \mathrm{Y}$ and ${ }^{96} \mathrm{Y}$ isotopes will be presented in the current paper. Details about the lifetime measurements of the inband states in the ${ }^{98} \mathrm{Y}$ nucleus will also be provided.

\section{Experimental details}

The data for the present study have been obtained from the two experimental campaigns conducted at the Institute Laue-Langevin in Grenoble. During the first one, named EXILL [5], the reactor neutron beam, with a flux of $10^{8} /\left(\mathrm{s} \times \mathrm{cm}^{2}\right)$, induced fission reactions $(\mathrm{n}, \mathrm{f})$ of the ${ }^{235} \mathrm{U}$ and ${ }^{241} \mathrm{Pu}$ targets. Gamma rays emitted from the fission product were detected by an array which consisted of eight HPGe clover detectors and 16 $\mathrm{LaBr}_{3}(\mathrm{Ce})$ FATIMA scintillators [6], mounted about 15 $\mathrm{cm}$ from the target position. The events were registered by using a fully digital acquisition system in a triggerless mode, with $100 \mathrm{MHz}$ clock. For the gamma spectroscopy measurement, the $\mathrm{LaBr}_{3}$ detectors where replaced by additional 6 large HPGe coaxial detectors and 2 clovers. The success of the EXILL campaign triggered the development of a permanent gammaspectroscopy experimental setup at ILL. The new array, called FIPPS, (FIssion Product Prompt $\gamma$-ray Spectrometer) [7] consists of up to $16 \mathrm{HPGe}$ clovers. For the new fission runs, ${ }^{233} \mathrm{U}$ and ${ }^{235} \mathrm{U}$ active targets have been used [8]. Scintillation light in the target cell provides precise information about the time when the

* Corresponding author: lukasz.iskra@mi.infn.it 
$(\mathrm{n}, \mathrm{f})$ reaction occurs, giving a clean fission tag. Data from the FIPPS campaign were partially sorted and included into the results presented in the current work.

\section{Data analysis and discussion}

Part of the analysis concerning lifetime measurements based on the fast-timing technique has been described, for the EXILL-FATIMA campaign, by J.-M. Régis et al. [9] and employed in order to study lifetimes of selected states in the ${ }^{90} \mathrm{Kr}$ and ${ }^{133} \mathrm{Sb}$ isotopes (see Refs [10] and [11], respectively). The method assumes sorting the data into triple coincidence events, where one $\gamma$-ray is collected by HPGe detector (with good energy resolution) and two gamma-rays by $\mathrm{LaBr}_{3}(\mathrm{Ce})$ scintillators (with good time resolution). The half-lives can be extracted from the difference between the centroids of the time distributions which correspond to the gamma rays feeding and decaying from the state of interest, if visible in the $\mathrm{LaBr}_{3}$ detectors [9]. The obtained results have to be corrected by using the prompt response difference (PRD) curve, as given in Ref. [9].

Further analysis has been performed for states in the ${ }^{98} \mathrm{Y}$ isotope [4]. The levels of interest are members of the rotational band built on the $4,6.95-\mu$ s isomer $[12,13]$. In order to measure the lifetime of the $5,596-\mathrm{keV}$ level, the $101-$ and $130-\mathrm{keV}$ transitions have been selected for the TAC "start-stop" combination. Whereas, the 186$\mathrm{keV}$ line has been used as gate in the HPGe array, providing a clean spectrum. During the analysis, various gating configurations have been used to avoid random coincidence event or contribution from other isotopes, when transitions with similar energy might occur. Figure 1 presents result of the analysis where the $T_{1 / 2}=175(25)$ ps value for the $5^{-}$state has been established. The analysis was continued upwards, along the rotational band, giving values of 51(10)- and 45(15)-ps half-lives for the $6^{-}$and $7^{-}$states, respectively. For the $8^{-}, 1070-\mathrm{keV}$ state the 15-ps upper limit has been obtained. The halflives which have been obtained during the analysis were used to calculate the reduced transition probabilities $\mathrm{B}(\mathrm{M} 1)$ and $\mathrm{B}(\mathrm{E} 2)$. Branching ratios of in-band transitions have been adopted from [14] and corrected for electron conversion coefficients. Therefore, the final deformation parameter for this rotational band was calculated as an average from the partial results, leading to $\beta=0.40(14)$.

The presence of a strongly deformed band in the $\mathrm{Y}$ nucleus with $\mathrm{N}=59$ encourages the search for rotational bands in lighter $\mathrm{Y}$ isotopes. Such an investigation has been performed for ${ }^{96} \mathrm{Y}$ and ${ }^{94} \mathrm{Y}$, using gamma spectroscopy methods. The data has been sorted into triple $\gamma$ coincidence cubes with various time windows. During the analysis also prompt-delayed gamma coincidences were used, where the time between two gammas corresponds to the half-lives of the isomers occurring in the studied isotopes. A detailed description of the coincidences techniques can be found in Refs. [14, $15]$.

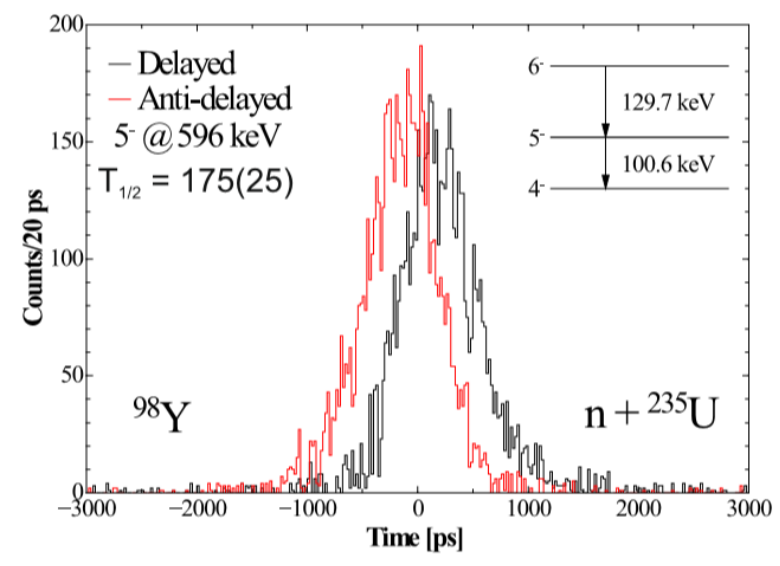

Fig. 1. TAC spectra of the corresponding distributions $D_{\text {stop }}$ (black) and $D_{\text {start }}$ (red) are shown

Laser spectroscopy studies of Ref. [17] provide information about the spherical character of the $0^{-}$ ground state and the $8^{+}$isomer in the ${ }^{96} \mathrm{Y}$ isotope. The latter observation has been confirmed in gamma spectroscopy study, by identifying the structure located above the isomer [18]. On the other hand, we propose that the new 201-ns state at $1655-\mathrm{keV}$ excitation energy is deformed [4], indicating the occurrence of shape coexistence in the ${ }^{96} \mathrm{Y}$ nucleus. This competition between shapes is even more pronounced in the heavier even-A Y isotopes, as it is shown in the pictorial view of Fig. 2. In the case of the ${ }^{100} \mathrm{Y}$ nucleus, a rotational band has already been observed on the $1^{+}, 11-\mathrm{keV}$ state and there is no sign of a spherical structure at higher spin values. Whereas, for the ${ }^{98} \mathrm{Y}, \mathrm{N}=59$ isotope, with 2 neutrons less, the presence of coexisting spherical and deformed shapes is very clear. In this case, the $0^{-}$ground state is spherical, as also confirmed by the laser spectroscopy study [17]. The deformation occurs at the higher excitation energy, around $0.5 \mathrm{MeV}$ where a significantly developed rotational band above the 6.95$\mu$ s isomer is found. In Ref. [17], also the second highly deformed isomer has been measured, later established at $466-\mathrm{keV}$ excitation energy by Urban et al. [13]. It is found that the overall feeding of the states with spherical and deformed configurations is more or less equal in the ${ }^{98} \mathrm{Y}$ isotope. This intensity balance is significantly unsettled in the ${ }^{96} \mathrm{Y}$ nucleus, with 2 neutrons less. Here the spherical configuration plays a dominant role, while the deformed structure is weak and moved even higher in excitation energy. The isomeric bandhead also has a shorter half-life in comparison to those in the ${ }^{98} \mathrm{Y}$ isotope. On the contrary, the spherical $8^{+}, 9.6-\mathrm{s}$ isomer in ${ }^{96} \mathrm{Y}$ is strongly fed, while the population of the shorter $10^{-}, 0.8-\mu \mathrm{s}$ counterpart in the heavier neighbor is relatively weak. 


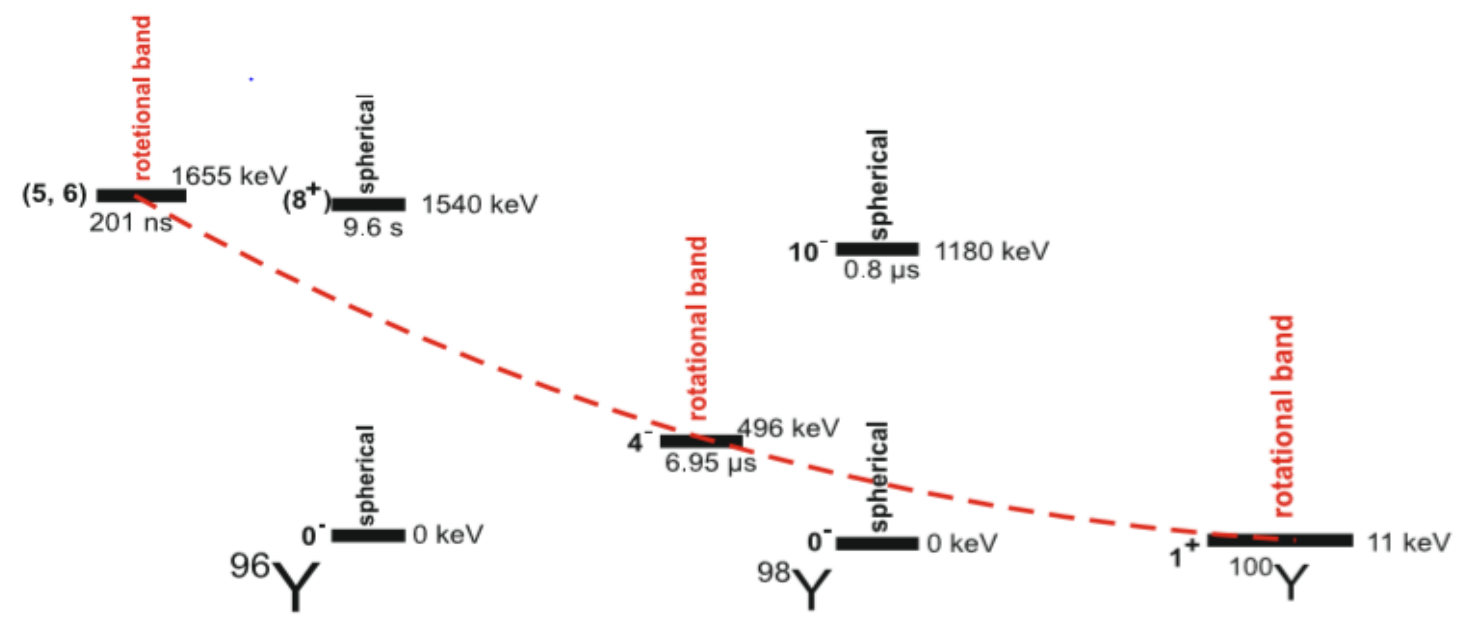

Fig. 2. Pictorial drawing of the evolution of deformed structures (in red) across the $Y$ isotopic chain

Inspired by the new results in the ${ }^{96} \mathrm{Y}$ isotope, our collaboration made an effort to develop the level scheme of the ${ }^{94} \mathrm{Y}$ nucleus, located 5 neutrons away from the $\mathrm{N}=$ 60 boundary. Using data from EXILL (and crosschecked with partially sorted data from FIPPS), the level scheme was extended up to $5 \mathrm{MeV}$ excitation energy [19], however, no evidence of deformed structure in this isotope was found. This is not surprising, taking into account the significant decrease in population of the rotational bands, in comparison with the spherical pattern when further away from $\mathrm{N}=60$. In addition, the fact that deformed structures move to higher excitation energy and isomeric bandheads disappear make even more difficult the identification of rotational bands in these nuclei.

\section{Conclusion}

The hypothesis that large deformation might occur also in yttrium isotopes with $\mathrm{N}<60$ is in line with recent results from the EXILL campaign, where, for the first time, a rotational band in $\mathrm{N}=57$ nucleus has been suggested [4]. According to this interpretation, deformed structures start to be present in lighter nuclei at high excitation energy and become dominant and yrast for isotopes with $\mathrm{N} \geq 60$. This scenario calls for further studies of the high-spin structures in the neutron-rich $\mathrm{Y}$ isotopes. The issue is being investigated taking advantage of new high quality and high statistic data from the FIPPS campaign, where a fission trigger based on an active scintillating target is used.

\section{Acknowledgments}

The authors acknowledge the technical services of the ILL for supporting the EXILL and FIPPS campaigns. This work was supported by the Italian Istituto Nazionale di Fisica Nucleare, and by the Polish National Science Centre under Contract No. 2014/14/M/ST2/00738 and 2013/08/M/ST2/00257.

\section{References}

1. K. Heyde and J. L. Wood., Rev. Mod. Phys., 83 1467 (2011).

2. W. U rban et al., N ucl. Phys A $\mathbf{6 8 9}, 605$ (2001).

3. T. W. Hagen et al., Phys. Rev. C 95, 034302 (2017).

4. Ł. W. Iskra et al., Europhys. Lett. 117, 12001 (2017).

5. M. J entschel et al., J. Instrum. 12, P11003 (2017).

6. FATIMA Technical Design Report and O. Roberts et al., N uc. Instr. M eth. 748, 91 (2014).

7. C. Michelagnoli et al., EPJ W eb Conf. 193, 04009 (2018).

8. F. Kandzia et al., in preparation

9. J.-M. Régis et al., Nucl. Instr. M eth. Phys. Res. A 763, 210 (2014).

10. J.-M. Régis et al., Phys. Rev. C 90, 067301 (2014).

11. G. Bocchi et al., Phys. Lett. B, 760273 (2016).

12. S. Brant, G. L hersonneau, and K. Sistemich, Phys. Rev. C 69, 034327 (2004).

13. W. Urban et al., Phys. Rev. C 96, 044333 (2017).

14. Balraj Singh and Zhiqiang Hu, NDS 98, 335 (2003).

15. Ł. W. Iskra et al., Phys. Rev. C89, 044324 (2014).

16. Ł. W. Iskra et al., Phys. Lett. B 788, 396 (2019).

17. B. Cheal et al., Phys. Rev. Lett. B 645, 133 (2007).

18. Ł. W. Iskra et al., A cta Phys. Pol. B 48, 581 (2017).

19. Ł. W. Iskra et al., Phys. Scr. 92, 104001 (2017). 